\title{
Does the approach influence the success rate for ultrasound-guided rectus sheath blocks? An anatomical case series
}

\author{
Ronald Seidel' \\ Andreas Wree ${ }^{2}$ \\ Marko Schulze ${ }^{2}$ \\ 'Department of Anesthesiology and \\ Intensive Care Medicine, HELIOS \\ Medical Center Schwerin, Schwerin, \\ ${ }^{2}$ Institute of Anatomy, Medical \\ University of Rostock, Rostock, \\ Germany
}

This article was published in the following Dove Press journal:

Local and Regional Anesthesia

5 July 2017

Number of times this article has been viewed

Correspondence: Ronald Seidel Department of Anesthesiology and Intensive Care Medicine, HELIOS Medical Center Schwerin, Wismarsche Strasse 393-7, 19049 Schwerin, Germany Tel +490385 520 425।

Email ronald.seidel@helios-kliniken.de

Andreas Wree

Institute of Anatomy, Medical University of Rostock, Gertrudenstrasse 9,

DE-18057 Rostock, Germany

Tel +49038 I 4948400

Email andreas.wree@med.uni-rostock.de

\begin{abstract}
The anterior cutaneous branches of the thoracolumbar spinal nerves enter the rectus sheath at its lateral border and perforate the muscle after travelling a short distance in the ventral direction. In this anatomical case series, we show that dye injection at the medial margin did not lead to reliable impregnation of the target nerves. The local anesthetic should therefore be injected at the lateral edge of the rectus sheath. Preceding surgical procedures to the ventral abdominal wall may affect the craniocaudal spread of local anesthetic.
\end{abstract}

Keywords: anesthesia, regional, ultrasonography, anatomic variation

\section{Basic anatomical knowledge}

The skin of the abdominal wall is innervated by the segments $\operatorname{Th} 7$ to L2, medially by the mammillary line through the anterior cutaneous branches and laterally by the mammillary line through the lateral cutaneous branches of the associated spinal nerves. ${ }^{1}$ These are mixed nerves, which also innervate the muscles of the abdominal wall.

The anterior cutaneous branches travel between the transversus abdominis and internal oblique abdominis muscle (Figure 1). They also branch and communicate widely within the transversus abdominis plane, especially along larger vessels such as the deep circumflex iliac artery (transversus abdominis plane plexus) and the epigastric arteries (rectus sheath plexus). Therefore, all nerves running along the anterior abdominal wall are mixed segmental nerves. ${ }^{2}$

The rectus abdominis muscle is innervated by the segments Th7 to Th12 (Th5 to L1). At the lateral edge of the rectus sheath, the anterior cutaneous branches divide into superficial (innervation of the skin) and deep branches, which enter laterally into the rectus sheath. From that point, they travel a short distance behind the rectus abdominis muscle before perforating ventrally and reaching the innervation area of the anterior abdominal wall. Within the rectus sheath, they give off branches to the posterior aspect of the rectus sheath and to the rectus abdominis muscle (Video S1: right side). This muscle is subdivided ventrally by tendinous intersections into several (usually three to four) segments. ${ }^{1}$ Therefore, local anesthetic must be applied posteriorly to the rectus abdominis muscle and ventrally to a clearly visible double layer in order to achieve a sufficient craniocaudal spread. This double layer consists of the aponeuroses of the transversus and the internal oblique abdominis muscles (Figure 1). 


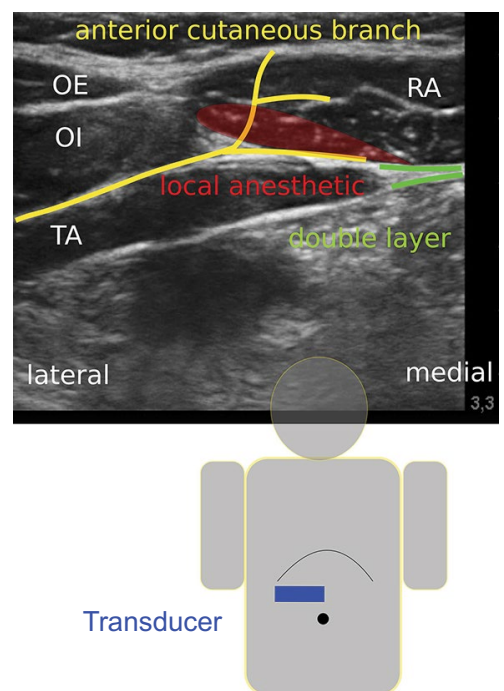

Figure I Transducer position, course of the anterior cutaneous branches (yellow) and instillation of local anesthetic (red).

Abbreviations: OE, external oblique muscle; OI, internal oblique muscle; TA, transversus abdominis muscle; RA, rectus abdominis muscle

\section{Methods}

The investigations were performed at the Institute of Anatomy, University of Rostock, Germany. Six ultrasoundguided rectus sheath blocks were performed on three unfixed donor cadavers, which had been designated for education or research, using $20 \mathrm{~mL}$ of Alcian blue.

The linear transducer ( $38 \mathrm{~mm}, 6-13 \mathrm{MHz}$ from Sonosite, Bothell, WA, USA) was placed in the transverse plane between the xiphoid and the umbilicus above the rectus abdominis muscle (Figure 1). The injections were performed using an in-plane technique either at the medial (in lateral direction) or at the lateral (in medial direction) edge of the rectus abdominis muscle with a SonoPlex ${ }^{\circledR}$ $21 \mathrm{G} \times 100 \mathrm{~mm}$ cannula from Pajunk, Geisingen, Germany. The ultrasound-optimized needle was placed between the rectus abdominis muscle and the posterior leaf (double layer) of the rectus sheath (Video S2: right side, injection from lateral to medial).

\section{Ethical statement}

This research was exempt from review by an ethics committee, because it did not include studies on living humans or animals.

\section{Results}

The results are summarized in Table 1. Injections at the lateral edge of the rectus sheath (first cadaver, right side [1R], second cadaver, right side [2R], third cadaver, left side [3L]) generally resulted in complete impregnation of the target nerves (Figure 2). An exception to this was observed with preparation 3R. In this case, the rectus abdominis muscle had lipomatous degeneration (as a result of partial denervation) following a conventional cholecystectomy and was adhered to the posterior leaf of the rectus sheath. Therefore, the spread of the dye was restricted in the cranial direction (Figure 3). Injections from medial to lateral did not result in the staining of the target nerves in both cases (1L and 2L) (Figure 4).

\section{Discussion}

Rectus sheath blockades are established analgesic procedures, especially in pediatric anesthesia. ${ }^{3,4}$ Recent studies show better postoperative analgesia for rectus sheath blocks compared to local wound infiltration for children undergoing umbilical hernia repair. ${ }^{5,6}$ In relation to adults, the target compartment is small and the local anesthetic may spread more evenly in the craniocaudal and mediolateral direction. However, current studies also describe the intraoperative performance of rectus sheath blocks by the surgeon. ${ }^{7,8}$

Table I Impregnation of the intercostal nerves (anterior cutaneous branches) inside the rectus sheath following the injection of $20 \mathrm{~mL}$ Alcian blue

\begin{tabular}{lll}
\hline Preparation & $\begin{array}{l}\text { Approach (in-plane) } \\
\text { from }\end{array}$ & $\begin{array}{l}\text { Staining of the anterior } \\
\text { cutaneous branches }\end{array}$ \\
\hline IR & Lateral & + \\
IL & Medial & - \\
2R & Lateral & + \\
2L & Medial & Incomplete ${ }^{\mathrm{a}}$ \\
3R & Lateral & Incomplete $^{\mathrm{a}, \mathrm{b}}$ \\
3L & Lateral & + \\
\hline
\end{tabular}

Notes: aln cranial direction. ${ }^{b}$ Condition after conventional cholecystectomy. Numbers I-3 refer to cadavers I-3.

Abbreviations: $L$, left side; $\mathrm{R}$, right side.

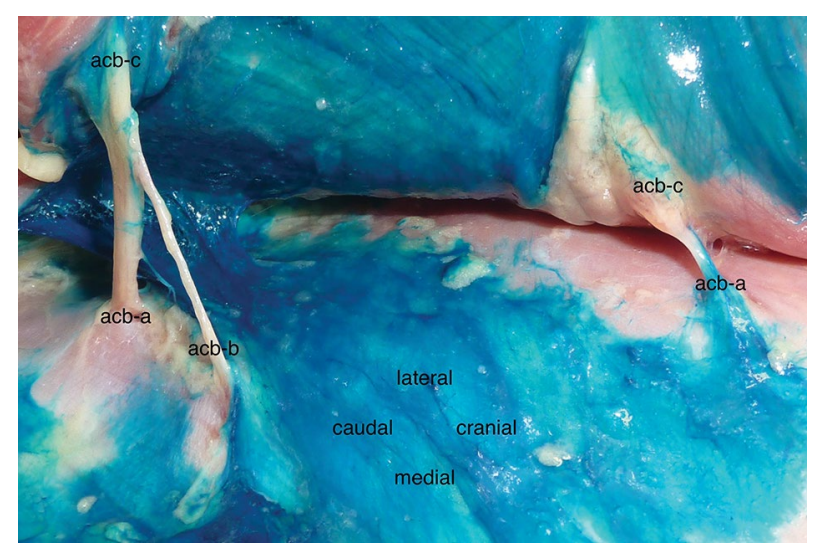

Figure 2 Anterior cutaneous branches (acb) impregnated by the dye (injection from lateral).

Notes: View from medial below the lateral edge of the rectus abdominis muscle. Preparation: first cadaver, right side (IR).

Abbreviations: acb-a, penetrating the posterior leaf of the rectus sheath; acb-b, giving off a branch to the posterior rectus sheath; acb-c, penetrating the rectus abdomins muscle. 


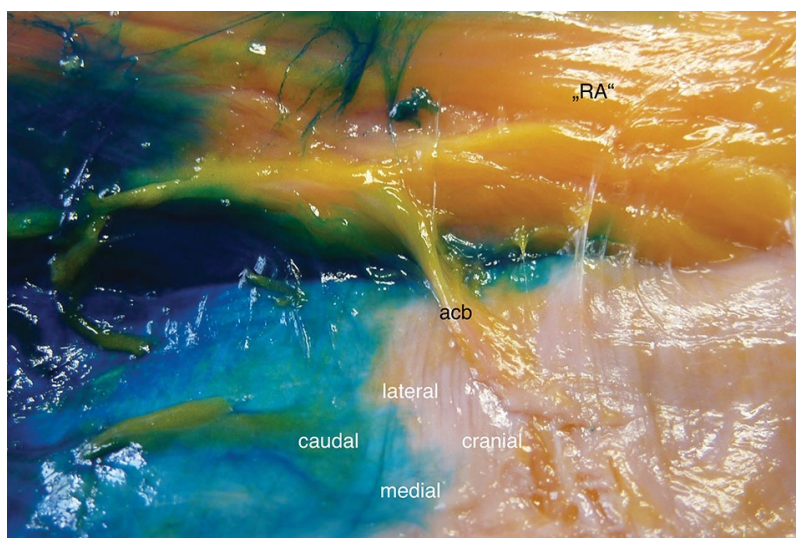

Figure 3 Lipomatous degeneration of the rectus abdominis muscle ("RA") and restricted spread of the injectant in the cranial direction.

Notes: View from medial below the lateral edge of the rectus abdominis muscle. Preparation: third cadaver, right side (3R).

Abbreviation: acb, anterior cutaneous branch.

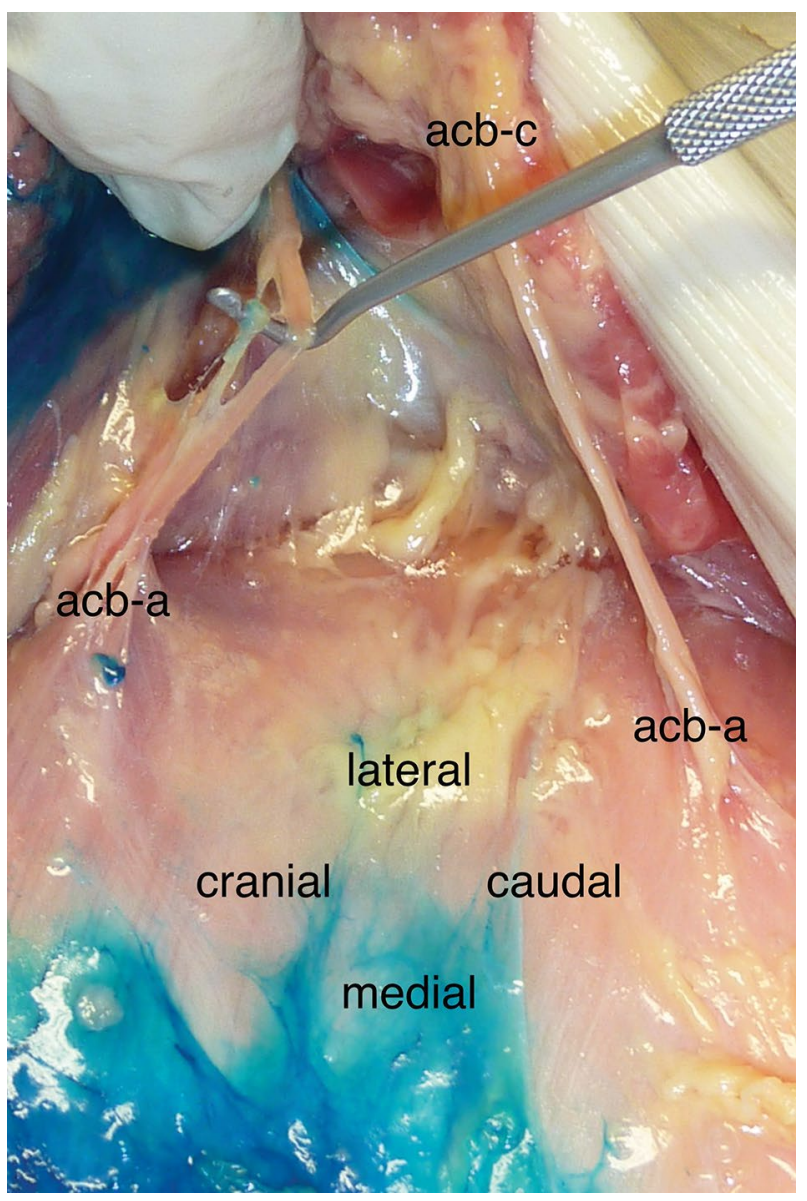

Figure 4 Anterior cutaneous branches (acb) without staining (injection from medial).

Notes: View from medial below the lateral edge of the rectus abdominis muscle. Preparation: first cadaver, left side (IL).

Abbreviations: acb-a, penetrating the posterior leaf of the rectus sheath; acb-c penetrating the rectus abdominis muscle.

In adults, this technique allows for a minor surgical procedure of the anterior abdominal wall without the need of additional general anesthesia (eg, minor revisions after midline laparotomy, umbilical hernia repair, or insertion of peritoneal dialysis catheters in high-risk patients). ${ }^{9}$ The sonoanatomic landmarks are clearly defined, and the spread of the injected local anesthetic can be visualized immediately. ${ }^{10} \mathrm{An}$ ultrasound-guided technique potentially avoids complications such as intraperitoneal injection or accidental punctures of the epigastric vessels. ${ }^{11}$

For truncal blocks, the duration of the blockade has been prolonged by continuous infusion or the use of liposomal bupivacaine in clinical trials or individual cases. ${ }^{12-14}$ These techniques can provide reasonable analgesia for selective major abdominal procedures when epidural anesthesia is contraindicated. Rectus sheath catheters can be placed in the craniocaudal or lateromedial direction without obstructing the sterile coverage of the surgical area. With regard to an improved or prolonged analgesic effect contradictory results were reported for the addition of dexmedetomidine or dexamethasone to local anesthetics. ${ }^{15-17}$ In the randomized study by Hamada et al, adding dextran to levobupivacaine decreased the risk of levobupivacaine toxicity (lower plasma concentrations) while providing better analgesia (lower resorption rate). ${ }^{18}$

Does the chosen approach influence the spread of the injectate and the success rate for rectus sheath blocks? Manassero et al examined the spread of local anesthetic in adult patients undergoing umbilical hernia repair. ${ }^{9}$ They performed the block ( $20 \mathrm{~mL}$ of levobupivacaine $0.375 \%$ + epinephrine $5 \mu \mathrm{g} / \mathrm{mL}$ ) at T10-level in a medial to lateral direction, with the aim to deposit the local anesthetic near the lateral border of the rectus sheath. Local anesthetic spread was evaluated ultrasonographically and scored from 0 to 4 (local anesthetic from T9- to T11-level, bilaterally). The overall success rate (no intraoperative supplementation with mepivacaine $1 \%$ at cutaneous incision) was $53.3 \%$. In patients with local anesthetic spread score $4(26.6 \%)$, the success rate was $75 \%$. The authors discuss two possible explanations for their results. First, the nerves within the rectus sheath are mixed segmental nerves, due to diverse anastomoses during their course along the anterolateral abdominal wall. ${ }^{2}$ This requires a larger craniocaudal spread of the injectate within the rectus sheath, which can be achieved by larger local anesthetic volumes, a multi-injection technique, or craniocaudal needle guidance. A second possible cause is aberrant cutaneous branches that are formed before the rectus sheath and run anterior to the rectus abdominis muscle in the subcutaneous tissue. ${ }^{1}$ These anatomical variants led to the concept of a para-umbilical block. ${ }^{19}$ Furthermore, a superiority of transversus abdominis plane (TAP) blocks to rectus sheath blocks could be postulated. For postoperative analgesia following umbilical hernia repair in children, the superiority of TAP blocks over rectus sheath blocks has not yet been proven. ${ }^{20}$ 
The range of possible regional anesthesia techniques for surgical interventions on the anterolateral abdominal wall is extensive, ranging from neuraxial blocks to local wound infiltration. ${ }^{21-27}$ These include the intravenous infusion of lidocaine (off-label use), selective nerve blocks (eg, iliohypogastric and ilioinguinal nerve), thoracic-paravertebral, ventral (eg, subcostal transversus abdominis plane and rectus sheath blocks), and dorsal fascial plane blocks. Newer methods (dorsal approach) such as the erector spinae plane and quadratus lumborum block (QLB) have yet to be validated in clinical and anatomical studies. A potential benefit could be the retrograde spread into the thoracic-paravertebral space, as shown in the cadaver study by Dam et al for the transmuscular QLB. This may expand the anesthetic field to include the sympathetic trunk. ${ }^{26,27}$

The choice of regional anesthesia technique for abdominal surgery is influenced by many factors. Previous operations on the abdominal wall, even outside of the rectus sheath, may compromise the spread of injected local anesthetic. In this situation, alternative procedures should be considered.

\section{Conclusion}

For ultrasound-guided rectus sheath blocks, the local anesthetic should be injected at the lateral edge of the rectus sheath, usually at the level of the surgical incision (due to the possibility of restricted craniocaudal spread). A lateral approach avoids the accidental puncture of the epigastric artery.

\section{Acknowledgment}

The Helios Research Centre provided financial support for the work.

\section{Disclosure}

The authors report no conflicts of interest in this work.

\section{References}

1. Loeweneck H, Feifel G. In: Lanz T von, Wachsmuth W, editors. Praktische Anatomie [Practical Anatomy]. Ein Lehr- und Hilfsbuch der anatomischen Grundlagen ärztlichen Handelns. Berlin: Springer-Verlag; 2004:33-57. German.

2. Rozen WM, Tran TM, Ashton MW, Barrington MJ, Ivanusic JJ, Taylor GI. Refining the course of the thoracolumbar nerves: a new understanding of the innervation of the anterior abdominal wall. Clin Anat. 2008;21(4):325-333.

3. de Jose Maria B, Götzens V, Mabrok M. Ultrasound-guided umbilical nerve block in children: a brief description of a new approach. Paediatr Anaesth. 2007;17(1):44-50.

4. Willschke H, Kettner S. Pediatric regional anesthesia: abdominal wall blocks. Paediatr Anaesth. 2012;22(1):88-92.
5. Dingeman RS, Barus LM, Chung HK, et al. Ultrasonography-guided bilateral rectus sheath block vs local anesthetic infiltration after pediatric umbilical hernia repair: a prospective randomized clinical trial. JAMA Surg. 2013;148(8):707-713.

6. Gurnaney HG, Maxwell LG, Kraemer FW, Goebel T, Nance ML, Ganesh A. Prospective randomized observer-blinded study comparing the analgesic efficacy of ultrasound-guided rectus sheath block and local anaesthetic infiltration for umbilical hernia repair. Br J Anaesth. 2011;107(5):790-795.

7. Litz CN, Farach SM, Fernandez AM, et al. Percutaneous ultrasoundguided vs. intraoperative rectus sheath block for pediatric umbilical hernia repair: a randomized clinical trial. J Pediatr Surg. In press 2017.

8. Landmann A, Visoiu M, Malek MM. Development of a novel technique for bilateral rectus sheath nerve blocks under laparoscopic-guidance. $J$ Pediatr Surg. In press 2017.

9. Manassero A, Bossolasco M, Meineri M, Ugues S, Liarou C, Bertolaccini L. Spread patterns and effectiveness for surgery after ultrasound-guided rectus sheath block in adult day-case patients scheduled for umbilical hernia repair. J Anaesthesiol Clin Pharmacol. 2015;31(3):349-353.

10. Webster K. Ultrasound-guided rectus sheath block - analgesia for abdominal surgery. Update in Anaesthesia. 2010;26:12-17.

11. Abrahams M, Derby R, Horn JL. Update on ultrasound for truncal blocks: a review of the evidence. Reg Anesth Pain Med. 2016;41(2):275-288.

12. Shido A, Imamachi N, Doi K, Sakura S, Saito Y. Continuous local anesthetic infusion through ultrasound-guided rectus sheath catheters. Can J Anaesth. 2010;57(11):1046-1047.

13. Tsui BC, Green JS, Ip VH. Ultrasound-guided rectus sheath catheter placement. Anaesthesia. 2014;69(10):1174-1175.

14. Hutchins JL, Kesha R, Blanco F, Dunn T, Hochhalter R. Ultrasoundguided subcostal transversus abdominis plane blocks with liposomal bupivacaine vs. non-liposomal bupivacaine for postoperative pain control after laparoscopic hand-assisted donor nephrectomy: a prospective randomized observer-blinded study. Anaesthesia. 2016;71(8):930-937.

15. Ammar AS, Mahmoud KM. Effect of adding dexamethasone to bupivacaine on transversus abdominis plane blocks for abdominal hysterectomy: a prospective randomized controlled trial. Saudi J Anaesth. 2012;6(3):229-233.

16. Ding W, Li W, Zeng X, et al. Effect of adding dexmedetomidine to ropivacaine on ultrasound-guided dual transversus abdominis plane block after gastrectomy. J Gastrointest Surg. In press 2017.

17. Huang SH, Lu J, Gan HY, Li Y, Peng YG, Wang SK. Perineural dexamethasone does not enhance the analgetic efficacy of ultrasound-guided subcostal transversus abdominis plane block during laparoscopic cholecystectomy. Hepatobiliary Pancreat Dis Int. 2016;15(5):540-545.

18. Hamada T, Tsuchiya M, Mizutani K, et al. Levobupivacaine-dextran mixture for transversus abdominis plane block and rectus sheath block in patients undergoing laparoscopic colectomy: a randomized controlled trial. Anaesthesia. 2016;71(4):411-416.

19. Courreges P, Poddevin F, Lecoutre D. Para-umbilical block: a new concept for regional anaesthesia in children. Paediatr Anaesth. 1997;7(3):211-214.

20. Torii N, Tachibana K, Iwasaki M, Takeuchi M, Kinouchi K. Ultrasoundguided rectus sheath block vs. transversus abdominis plane block in children undergoing umbilical hernia repair. Masui. 2016;65(6):560-565.

21. Børglum J, Gögenür I, Bendtsen TF. Abdominal wall blocks in adults. Curr Opin Anaesthesiol. 2016;29(5):638-643.

22. El-Boghdadly K, Madjdpour C, Chin KJ. Thoracic paravertebral blocks in abdominal surgery - a systematic review of randomized controlled trials. Br J Anaesth. 2016;117(3):297-308.

23. Hebbard PD. Transversalis fascia plane block, a novel ultrasound-guided abdominal wall nerve block. Can J Anaesth. 2009;56(8):618-620.

24. Hebbard PD, Barrington MJ, Vasey C. Ultrasound-guided continuous oblique subcostal transversus abdominis plane blockade: description of anatomy and clinical technique. Reg Anesth Pain Med. 2010;35(5): 436-441. 
25. Weibel S, Jokinen J, Pace NL, et al. Efficacy and safety of intravenous lidocaine for postoperative analgesia and recovery after surgery: a systematic review with trial sequential analysis. Br JAnaesth. 2016;116(6):770-783.

26. Chin KJ, Adhikary S, Sarwani N, Forero M. The analgesic efficacy of pre-operative bilateral erector spinae plane (ESP) blocks in patients having ventral hernia repair. Anaesthesia. 2017;72(4):452-460.
27. Dam M, Moriggl B, Hansen CK, Hoermann R, Bendtsen TF, Børglum $\mathrm{J}$. The pathway of injectate spread with the transmuscular quadratus lumborum block: a cadaver study. Anesth Analg. In press 2017.

Local and Regional Anesthesia

\section{Publish your work in this journal}

Local and Regional Anesthesia is an international, peer-reviewed, open access journal publishing on the development, pharmacology, delivery and targeting and clinical use of local and regional anesthetics and analgesics. The journal is included in PubMed, and welcomes submitted papers covering original research, basic science, clinical studies,

\section{Dovepress}

reviews and evaluations, guidelines, expert opinion and commentary, case reports and extended reports. The manuscript management system is completely online and includes a very quick and fair peer-review system, which is all easy to use. Visit http://www.dovepress.com/ testimonials.php to read real quotes from published authors. 